\title{
La educación rural en Colombia: experiencias y perspectivas
}

\author{
Martha Lucia Carrero Arango ${ }^{1}$ \\ Universidad Pedagógica Nacional, Bogotá, Colombia. \\ martha.carrero17@gmail.com \\ María Fernanda González Rodríguez ${ }^{2}$ \\ Universidad Pedagógica Nacional, Bogotá, Colombia \\ gonzalezmafe01@gmail.com
}

1 Estudiante de la Licenciatura en Diseño Tecnológico, UPN, Bogotá D.C.

2 Estudiante de la Licenciatura en Diseño Tecnológico, UPN, Bogotá D.C. 


\section{La educación rural en Colombia: experiencias y perspectivas}

\section{Resumen}

El presente texto presenta una reflexión sobre la situación de la educación rural en Colombia, sus aportes a la educación, su relación con la educación en tecnología y algunas experiencias frente a la educación rural en la localidad de Ciudad Bolívar.

Palabras claves: Educación rural, modelos pedagógicos, tecnología

\section{Rural education in Colombia: experiences and perspectives}

\section{Abstract}

This article presents a reflection about the situation of the rural education in Colombia, its contributions to the education, its relation with the education in technologies and some educational experiences in the municipality of Ciudad Bolívar.

Keywords: rural education, pedagogical models, technology.

\section{A educação rural na Colômbia: experiências e perspectivas}

\section{Resumo}

O presente texto apresenta uma reflexão sobre a situação da educação rural na Colômbia, suas contribuições à educação, sua relação com a educação em tecnologia e algumas experiências em frente à educação rural na localidade de Cidade Bolívar.

Palavras-chave: Educação rural, modelos pedagógicos, tecnologia 


\section{Introducción}

El campo colombiano durante más de cuatro décadas ha sido escenario de violencia, pobreza y reformas fallidas o inconclusas. El $94 \%$ del territorio del país es rural, el $32 \%$ de la población vive allí y las condiciones educativas de esta población son precarias. Aunque no ha existido una política educativa coherente con estos sectores, el Estado se ha preocupado por resolver algunos problemas parciales de la educación rural colombiana.

El artículo aborda los siguientes aspectos: en primer lugar, se enuncian algunos aspectos históricos de la educación rural en Colombia, el trayecto de ésta durante la época de conflicto — década de 1950 - hasta hoy; en segundo lugar, se hace un esbozo de los modelos educativos alternativos formulados por el Ministerio de Educación Nacional — MEN — que dan respuesta a las necesidad de la población rural; acto seguido, se hace referencia a la relación educación en tecnología y educación rural; posteriormente se mencionan experiencias frente a la educación rural en la localidad de Ciudad Bolívar; $y$, finalmente, se enuncian las perspectivas que se han construido frente a la educación rural.

\section{La educación rural en Colombia}

Los términos indígena y campesino han sido vistos como sinónimos de inferioridad, de marginalidad y pobreza. Esto constituye un accidente cultural que ha venido acompañándonos desde hace más de cinco siglos. Si habláramos de lo rural también existiría esa connotación, pues como colombianos hemos olvidado que los ciudadanos no solamente somos los que vivimos en la ciudad, sino que el campo es parte esencial del desarrollo de las ciudades. Tal olvido refleja el gran desequilibrio que ha existido entre lo urbano y lo rural; la educación no es la excepción.

En el medio rural, el servicio educativo se ha visto influido por las condiciones socio-económicas, culturales y de infraestructura de las poblaciones rurales. En términos generales, se puede describir una escuela en el medio rural como un establecimiento pobre, estropeado, con poca dotación y mobiliario poco funcional, condiciones que hacen que sean vistas como poco interesantes y de ese mismo modo el Estado y las políticas olvida el medio rural.

El auge de la educación rural se inicia en la década de 1970 cuando el Gobierno Nacional incorpora la educación a las políticas de reforma agraria y de desarrollo rural para promover el cambio social. Pero, ¿qué es educación? Tomando lo dicho por Lynn Smith, éste señala que: 
Educación es el nombre aplicado al proceso mediante el cual la parte socialmente aprobada de la herencia cultural se transmite de una generación a otra, y el proceso mediante el cual el conocimiento recién adquirido se difunde entre los miembros de la sociedad (1960, p. 413).

De ahí que la educación haga parte de las actividades principales de la sociedad, razón por la cual debe estar presente para cada sector de la ciudad; claro está que la educación se imparte, en primer lugar, por la familia; en segundo lugar, por la escuela y, como tercer lugar, según lo dicho por Lynn Smith, tenemos el servicio de extensión (1960), el cual opera en los colegios de agricultura y tiene como objetivo el mejoramiento de la vida de los pobladores rurales.

La educación en Colombia ha sido definida como un derecho constitucional. En la Ley 115 de 1994 — Ley General de Educación_- en el artículo 1 se menciona que: «La educación es un proceso de formación permanente, personal, cultural y social que se fundamenta en una concepción integral de la persona humana, de su dignidad, de sus derechos y de sus deberes»; y hace la división entre educación formal, no formal e informal a saber:

La educación formal es aquella que se imparte en establecimientos educativos aprobados, en una secuencia regular de ciclos lectivos, con sujeción a pautas curriculares progresivas, y conducentes a grados y títulos. (Ley 115 de 1994, art. 10)

La educación no formal se ofrece con el objeto de complementar, actualizar, suplir conocimientos y formar en conocimientos y aspectos académicos o laborales sin sujeción al sistema de niveles y grados establecidos para la educación formal. (Art. 36)

Se considera educación informal todo conocimiento libre y espontáneamente adquirido, proveniente de personas, entidades, medios masivos de comunicación, medios impresos, tradiciones, costumbres, comportamientos sociales y otros no estructurados. (Art. 43)

Aparece luego, en el capítulo cuarto de la Ley, la educación campesina y rural, sobre la que se señala que:

El Gobierno Nacional y las entidades territoriales promoverán un servicio de educación campesina y rural la cual comprenderá especialmente la formación técnica en actividades agrícolas, pecuarias, pesqueras, forestales y agroindustriales que contribuyan a mejorar las condiciones humanas, de trabajo y la calidad de vida de los campesinos y a incrementar la producción de alimentos en el país. (Ley 115 de 1994, art. 64)

La educación rural hoy por hoy ha adquirido mayor importancia en la sociedad debido a la superación de problemas que aquejan al territorio, en nuestro caso, 
al sector educativo. Dentro de los problemas que afectan al sistema educativo se ubicaba la baja cobertura, la falta de calidad y la pertinencia de una política educativa que responda a las necesidades sociales del contexto. Con miras a la construcción e implementación de herramientas que den solución a los problemas ya mencionados, el Ministerio de Educación Nacional implementó el Proyecto de Educación Rural —PER-, que en su Fase II tiene como objetivo:

[...] incrementar el acceso con calidad a la educación en el sector rural desde preescolar hasta media, promover la retención de niños, niñas y jóvenes en el sistema educativo y mejorar la pertinencia de la educación para las comunidades rurales y sus poblaciones escolares con el fin de elevar y la calidad de vida de la población rural. (MEN, 2013)

\section{Modelos educativos alternativos}

En cuanto a las orientaciones que el docente que se encuentra en el ámbito rural debe implementar, el Ministerio de Educación Nacional formula los modelos pedagógicos flexibles, los cuales son propuestas de educación formal que permiten atender a poblaciones diversas o en condiciones de vulnerabilidad que presentan dificultades para participar en la oferta educativa tradicional. Estos modelos se caracterizan por tener una propuesta educativa acorde a las limitaciones que tienen dichas poblaciones.

A continuación, haremos un breve recuento de los modelos que están contemplados en la actual política educativa rural:

a. Aceleración del aprendizaje: este modelo busca apoyar a niños, niñas y jóvenes de básica primaria que están en extra edad, con miras a mejorar su potencial de aprendizaje, a que estos permanezcan en las escuelas y así avancen y culminen sus estudios.

b. Posprimaria: este modelo educativo es pensado para ser desarrollado a partir de la formulación de proyectos pedagógicos productivos y mediante el uso de guías de aprendizaje.

c. Telesecundaria: igualmente este modelo busca que los niños, niñas y jóvenes continúen con su proceso formativo, pero a diferencia de los ya mencionados, centra sus estrategias en la televisión educativa y en modelos de aprendizaje en el aula.

d. Servicio de Educación Rural - SER-: modelo educativo que, partiendo de la realidad y las potencialidades que existen en cada comunidad, define sus líneas de trabajo y núcleos temáticos para la integración de las diferentes áreas del saber.

e. Programa de Educación Continuada Cafam: es un programa que piensa la 
educación como estrategia para el desarrollo humano de jóvenes y adultos, base del desarrollo social y económico.

\section{Sobre la tecnología y la educación rural}

¿Cómo la incorporación de la tecnología fortalece los procesos políticos, sociales, económicos, culturales y educativos en el área rural, de tal modo que supla las necesidades reales de ésta? Con la intención de dar respuesta a lo mencionado, inicialmente se hará un breve recuento del sector educativo en el contexto rural seguido de la descripción de la observación de experiencias de integración y formación del área tecnológica en la educación, utilizando como elemento complementario algunos de los componentes que tiene el PER —en relación a propuestas pedagógicas que éste contiene-, para finalizar con la reflexión y concepción que se tiene sobre la enseñanza del área tecnológica.

Abordaremos el tema de la educación para la población rural en Colombia trayendo a colación dos aspectos a desarrollar: uno es la integración que ha tenido la educación en el contexto rural, y dos la implementación del modelo pedagógico de la Escuela Nueva.

Haciendo un esbozo del primer aspecto, la Ley General de Educación define la educación como un derecho constitucional en el artículo 1 del cual ya hemos hecho mención, razón por la cual el Estado se ve obligado a pensar de qué forma implementar políticas educativas acordes a la zona rural sin olvidar, claro está, que en medio de esta zona han acontecido un sinnúmero de acciones que alteran la estructura educativa. Es preciso anotar que dentro de los factores con mayor peso encontramos el incremento del conflicto armado, situación que conllevó, desde el punto de vista educativo, a la suspensión parcial o definitiva de actividades escolares. Superada esta situación, en la década de 1990 se muestra un balance positivo con respecto al aumento del alfabetismo y la escolaridad promedio de la población gracias a la expansión y consolidación de modelos educativos concebidos para este espacio, como el caso de la Escuela Nueva. (Perfetti, 2003)

La Escuela Nueva fue creada en 1975 como respuesta a los persistentes problemas de la educación rural colombiana y buscó introducir nuevas estrategias operativas que permitieran una mayor viabilidad técnica, política y financiera (Colbert, 1999), de allí que, como recurso educativo, es el más adecuado pues se caracteriza por ser un modelo mucho más flexible en el que se evidencia el trabajo en equipo, se observa una intervención personalizada y colaborativa por parte del docente y se implementa el uso y desarrollo de materiales educativos que ayudan en la formación del alumno. Esto permite la creación de lazos con la comunidad a través de proyectos de desarrollo local, aspectos que son importantes, ya que fueron los que dieron pie para que el Ministerio de Educación diseñara e implantara el Proyecto 
La educación rural en Colombia: experiencias y perspectivas

| Martha Lucia Carrero Arango | María Fernanda González Rodríguez |

de Educación Rural —PER-, en el cual se desarrollan estrategias pedagógicas que mejoren las prácticas de enseñanza y aprendizaje en docentes y estudiantes del campo, considerando la multiplicidad de condiciones propias de la ruralidad. Aquí es donde se crean los Proyectos Pedagógicos Productivos — ppp-. Estos proyectos se desarrollan como estrategia para facilitar la adquisición de competencias básicas y laborales generales sobre la base de experiencias de aprendizaje en contextos reales de producción con enfoque ambiental, buscando no sólo mejorar los aprendizajes sino crear un vínculo entre el sistema educativo y el sistema productivo nacional, lo que fomenta la cultura del emprendimiento en el campo. (MEN, 2013)

Entonces, ¿de qué forma entraría la enseñanza de la tecnología en la educación rural si es evidente que la tecnología ha sido una de las grandes olvidadas en los currículos? Bajo la pretensión de vincular la tecnología al Proyecto de Educación Rural, es necesario explicar qué se entiende por tecnología y por enseñanza de la tecnología.

Según las Orientaciones Generales para la Educación en Tecnología del Ministerio de Educación Nacional, la tecnología es una:

[...] actividad humana, la tecnología busca resolver problemas y satisfacer necesidades individuales y sociales, transformando el entorno y la naturaleza mediante la utilización racional, crítica y creativa de recursos y conocimientos. Otros aspectos igualmente importantes son el conocimiento y los procesos necesarios para crear y operar esos productos, tales como la ingeniería del saber cómo y el diseño, la experticia de la manufactura y las diversas habilidades técnicas.

Consideremos ahora lo que es la enseñanza de la tecnología, pero antes hay hacer mención de tres consideraciones o tendencias que se tienen de la tecnología. En primer lugar, se hace uso de la tecnología como medio de formación y reconocimiento práctico con un oficio específico; la segunda consideración es una concepción de tecnología como servidor de la ciencia y dependiente de ella, en la que la ciencia da explicación a las implicaciones que tiene la tecnología; y la tercera y última, radica en la tecnología como una actividad teórica-práctica apoyada en procesos de reflexión-acción (Rodríguez, 1998). Relacionando lo dicho hasta el momento, la Educación en Tecnología da la posibilidad al estudiante en su formación de afrontar competencias en su vida por medio del manejo de fuentes de información y el desarrollo de capacidades para la presentación de propuestas de solución de problemas vinculados a necesidades concretas.

Quizá no exista, estrictamente hablando desde el punto de vista legal, una correlación entre la educación en tecnología y la educación rural, sin embargo, podemos entender la tecnología no sólo como un área del conocimiento que se da en la escuela, sino como una herramienta que busca contrarrestar el aislamiento al que muchas escuelas rurales se ven sometidas, posibilitar proyectos comunes entre 
escuelas del mismo municipio o vereda, compartir recursos, favorecer el desarrollo del pensamiento tecnológico y fortalecer la relación comunidad-escuela por medio de proyectos agropecuarios, agroindustriales o ecológicos. De igual manera la tecnología abordaría temas encaminados hacia lo siguiente: una mirada reflexiva sobre la técnica, la cual se encuentra sujeta a los intereses de la comunidad; ser el agente motivador de un aprendizaje significativo; tener presente el constante cambio en el que se encuentra la sociedad de tal forma que responda a las circunstancias de cambio de la vida diaria y al desarrollo de conocimiento; su relación con la ciencia y otras fuentes del saber. Por tanto, la tecnología será flexible para adecuar sus contenidos y métodos de enseñanzas (Guillén, 2006), consideraciones que responden a las características establecidas en el modelo pedagógico de Escuela Nueva que se implementa en la educación rural.

\section{Algunas experiencias frente a la educación rural}

En el ámbito rural encontramos profesores que, si bien su formación no estuvo enmarcada dentro de la educación rural, se han esforzado por trabajar en beneficio de las poblaciones rurales, han estudiado y transformado su quehacer al contexto, haciendo de la escuela una escuela más activa, es decir, una escuela abierta, democrática y participativa, de las cuales tuvimos un acercamiento y haremos un breve recuento.

\section{Acercamiento a la educación rural: localidad Ciudad Bolívar}

Ciudad Bolívar es la localidad número 19 de Bogotá y cuenta con una extensión rural Ilamada Pasquilla. Esta vereda cuenta con una institución educativa Ilamada IED Pasquilla, la cual tiene tres sedes: sede A, Pasquilla; sede B, Santa Bárbara; y sede C, Pasquillita. La visita a este sector tuvo como intención contar a nuestros lectores el quehacer del docente y el modelo pedagógico por el que se rige cada sede.

\section{Sede Santa Bárbara}

Con respecto al quehacer docente, en esta sede se debe hacer mención de que el modelo por el que ésta se rige es el de Escuela Nueva y un sólo docente atiende a toda la primaria. Los grados que maneja la sede oscilan de grado cero a quinto y cuenta con aula de informática, una escuela de padres, huerta escolar y proyecto escolar.

La profesora encargada se llama Luz Marina, es Licenciada en Educación Preescolar y lleva en esta sede diez años. Actualmente la sede Santa Bárbara cuenta con 22 niños, de los cuales cinco cursan el grado cero; tres el grado primero; cinco el grado segundo; tres el grado tercero; uno el grado cuarto; y tres el grado quinto. A pesar de que esta sede es la más lejana, la docente muestra gran entusiasmo por 
La educación rural en Colombia: experiencias y perspectivas

| Martha Lucia Carrero Arango | María Fernanda González Rodríguez |

la labor que desempeña allí, ya que considera que estar y ser parte de la crianza de los niños del área rural es importante; igualmente menciona que el acompañamiento por parte de los padres en el desarrollo de actividades ha generado espacios de acercamiento y de trabajo colectivo para el avance y progreso de los estudiantes.

\section{Sede Pasqullita}

En cuanto a esta sede el trabajo con los niños se rige bajo el aula integrada. El aprendizaje va dirigido bajo tres ritmos y cada estudiante tiene atención personalizada. En Pasquillita se encuentran cuatro profesores y en cada intervención se recoge el amor que sienten al estar y ser parte de la comunidad rural, aunque llama la atención lo dicho por el profesor Samuel Orozco — profesor que ganó el Premio Compartir al Maestro-, quien menciona que nosotros como futuros profesores debemos dejar de lado el miedo al cambio, que somos nosotros los que sembramos en cada estudiante la semilla de la libertad y los que luchan por que cada niño y niña pueda ser un único ser integral, dejando en claro que se debe tener presente el papel que desempeña el docente en lo rural y en lo urbano. Tanto el docente rural como el urbano deben interpretar el contexto en el que se encuentran y no sólo deben cumplir con la labor pedagógica, sino debe ser y sentirse parte de la comunidad.

\section{Sede Pasquilla}

En esta sede se encuentra parte de la primaria y el bachillerato, cuenta con 15 docentes sumando las jornadas tarde y mañana. Debido a que no se tuvo un acercamiento con docentes de esta sede, la persona que se encuentra acompañando el recorrido y los estudiantes guías cuentan que los docentes en esta sede se preocupan por que cada estudiante alcance un desarrollo cognitivo óptimo, sembrando en ellos el amor por el campo, lo cual se evidenció en la finca del colegio donde los estudiantes realizan cada actividad con plena libertad y seguridad.

De esta forma la planta docente de la Institución Educativa Pasquilla le apuesta a la construcción de ciudadanos pacíficos, críticos y propositivos, conscientes de su entorno, que mejoren sus habilidades académicas y, sobre todo, crezcan como seres humanos autónomos, bajo el conocimiento de ser parte de una comunidad y ser facilitadores de mejores condiciones de vida. Las actividades de recolección y venta de desechos sólidos, de reforestación, de labores agropecuarias orgánicas, de producción de texto a partir de lo que se hace en el campo, les permite acercarse a su comunidad y a su contexto. Por todo esto, se puede decir que la clave está en la posibilidad de generar un aprendizaje experiencial a partir del desarrollo de proyectos que integran a toda la comunidad educativa, de igual manera es evidente cómo esta institución responde de manera asertiva a los lineamientos y estándares que plantea la Ley General de Educación identificando claramente las limitaciones y potencialidades de una zona rural. 


\section{Perspectivas para la educación rural}

Es importante enmarcar la situación de la educación rural dentro de la estructura económica y social para poder estudiar los mecanismos pertinentes que logren articular las necesidades educativas de los sectores con los servicios educativos disponibles, diseñados y pensados para los mismos.

Ahora bien, como docentes en formación se hace necesario pensar estrategias que aporten al mejoramiento y avance de la educación rural, además se hace indispensable que desde todos los sectores educativos se hagan aportes a la educación rural para poder eliminar la concepción de lo subordinado y lo marginado, pues es parte de nuestra cultura e identidad patrimonial.

Asimismo, es imperativo superar la concepción sectorial de lo educativo para integrar a plenitud políticas educativas que no sólo beneficien los centros urbanos, sino a los sectores rurales y, de ese modo, se amplíe la cobertura, la calidad y se tenga en cuenta el contexto específico de los sitios. 


\section{Referencias}

Colbert, V. (1999). Mejorando el acceso y la calidad de la educación para el sector rural pobre. El caso de la Escuela Nueva en Colombia. Revista Iberoamericana de Educación (20), 107-135.

Guillén, D., \& Santamaría, O. (2006). La enseñanza de la Tecnología en la Educación Básica (Un enfoque pedagógico). I Congreso Iberoamericano de Ciencia, Tecnología, Sociedad e Innovación CTS+l (pp. 1-11). México D.F.: OEI.

Ministerio de Educación Nacional —MEN. (2013). Mineducación. Recuperado el 25 de octubre de 2015, de Proyecto de Educación Rural PER: http://www.mineducacion.gov. co/1759/w3-article-329722.html

Perfetti, M. (2003). Estudio sobre la educación rural en Colombia. Recuperado el 6 de abril de 2016, de Red Latinoamericana de Educación Rural: red-ler.org/estudio_educacion_ poblacion_rural_colombia.pdf

Rodríguez, G. (1998). Ciencia, Tecnología y Sociedad: una mirada desde la Educación en Tecnología. Revista Iberoamericana de Educación(18), 107-143.

Smith, L. (1960). Sociología de la vida rural. Buenos Aires: Bibliográfica Argentina.

Recibido: 4 febrero 2016

Aceptado: 5 marzo 2016

Cómo citar: Carreri, M y González, M. (2016). La educación rural en

Colombia: experiencias y perspectivas. Praxis Pedagógica, 19, 79-89 